\title{
E-CHARACTERISTIC POLYNOMIALS OF TENSORS*
}

\author{
AN-MIN LI ${ }^{\dagger}$, LIQUN QI ${ }^{\ddagger}$, AND BIN ZHANG $\$$
}

\begin{abstract}
In this paper, we show that the coefficients of the E-characteristic polynomial of a tensor are orthonormal invariants of that tensor. When the dimension is 2 , some simplified formulas of the E-characteristic polynomial are presented. A resultant formula for the constant term of the E-characteristic polynomial is given. We prove that both the set of tensors with infinitely many eigenpairs and the set of irregular tensors have codimension 2 as subvarieties in the projective space of tensors. This makes our perturbation method workable. By using the perturbation method and exploring the difference between E-eigenvalues and eigenpair equivalence classes, we present a simple formula for the coefficient of the leading term of the E-characteristic polynomial when the dimension is 2 .
\end{abstract}

Key words. E-eigenvalues, tensors, E-characteristic polynomials, eigenpair equivalence class, irregularity.

AMS subject classifications. $65 \mathrm{H} 17$.

\section{Introduction}

Eigenvalues of higher order tensors were introduced in $2005[15,13]$ and have attracted much attention in the literature and found applications in science and engineering. The E-eigenvalues of a tensor were introduced in [15, 17]. E-eigenvalues are invariant under orthonormal coordinate changes [17]. They have applications in determining positive definiteness of a multivariate system [15, 19], best rank-one approximation $[15,18,19,24]$, magnetic resonance imaging $[5,20]$, spectral hypergraph theory [9] and symmetric embedding [21], and possess links with geometry $[1,2,3,4,16]$. The numbers of E-eigenvalues and E-eigenvectors have been studied in $[7,14]$.

An $n$-dimensional $m$ th order hypermatrix $A$ with entries in $\mathbb{C}$ is a map from $\{1, \cdots, n\}^{m} \rightarrow \mathbb{C}$. We use notation $A=\left(a_{i_{1} i_{2} \cdots i_{m}}\right)$ to denote such a hypermatrix.

In physics [11, 22], a tensor is a physical quantity. In geometry, a tensor can be regarded as a multi-linear function in a Hilbert space; see the discussion in Section 3. In such applications, in an orthonormal coordinate system, a tensor is expressed by a hypermatrix. It is important to determine parameters and properties of such a tensor, which are invariant under orthonormal coordinate changes. In Theorem 1 of Section 3 of this paper, we show that the coefficients of the E-characteristic polynomial of a tensor are orthonormal invariants of that tensor. For that discussion, we need to distinguish a tensor $\mathcal{A}$ and its hypermatrix $A$ in a coordinate system.

For some other applications, such as determining positive definiteness of a multivariate system, best rank-one approximation, spectral hypergraph theory, and symmetric embedding, there are no coordinate systems involved. People just regard a Jin.

*Received: February 22, 2011; accepted (in revised form): April 13, 2012. Communicated by Shi

This work is supported by the Research Grant Council of Hong Kong, Project Numbers: PolyU 501808,501909 , and 502510 .

${ }^{\dagger}$ School of Mathematics, Sichuan University, Chengdu, 610064, P.R. China (math_li@yahoo.com.cn).

${ }^{\ddagger}$ Department of Applied Mathematics, The Hong Kong Polytechnic University, Hung Hom, Kowloon, Hong Kong, P.R. China (maqilq@polyu.edu.hk).

$\S$ School of Mathematics, Sichuan University, Chengdu, 610064, P.R. China (zhangbin@scu.edu.cn). 
hypermatrix $A$ as a tensor. Hence, in the other parts of this paper, a tensor simply means a hypermatrix.

For an $m$ th order tensor $A$ and a vector $x \in \mathbb{C}^{n}$, we define $A x^{m-1}$ to be the vector in $\mathbb{C}^{n}$ with its $i$ th component as

$$
\left(A x^{m-1}\right)_{i}=\sum_{i_{2}, \cdots, i_{m}=1}^{n} a_{i i_{2} \cdots i_{m}} x_{i_{2}} \cdots x_{i_{m}}
$$

If $\lambda \in \mathbb{C}$ and $x \in \mathbb{C}^{n}$ satisfy

$$
\left\{\begin{array}{c}
A x^{m-1}=\lambda x \\
x^{T} x=1
\end{array}\right.
$$

then $\lambda$ is an E-eigenvalue of $A$ and $x$ is an E-eigenvector of $A$. If $A$ and $x$ are real, then $\lambda$ is also real [15]. In this case, $\lambda$ is called a Z-eigenvalue of $A$ and $x$ is called a Z-eigenvector of $A$. Z-eigenvalues play the main role in the above-mentioned applications. Algorithms for finding Z-eigenvalues were studied in [12, 19].

E-eigenvalues and Z-eigenvalues are invariant under orthonormal coordinate changes [15]. An even-order real symmetric tensor always has Z-eigenvalues [15]. It is positive definite (semi-definite) if and only if all of its Z-eigenvalues are positive (nonnegative) [15]. The Z-eigenvalue with the largest absolute value and its corresponding Z-eigenvector form the best rank-one approximation of a real symmetric tensor $[15,19]$. The modifier "E-" stands for Euclidean, as (1.1) implies that the Euclidean norm of $x$ is 1 if $x$ is real. The modifier "Z-" names after Prof. Shuzi Zhou, who suggested (1.1) to the author of [15]. Prof. Zhou departed by cancer in 2009. The name "Z-eigenvalue" has already been used widely in the literature $[1,2,3,4,5,7,9,12,14,15,16,17,18,19,20,23,24]$. Hence, we continue to use this name.

E-characteristic polynomials were introduced in [15, 17], and discussed in $[14,6]$. Until now, there are no other papers containing a discussion on E-characteristic polynomials. We now survey the definitions and properties of E-characteristic polynomials in $[6,14,15,17]$

Definition 1.1. Let $A=\left(a_{i_{1} i_{2} \cdots i_{m}}\right)$ be an $n$-dimensional mth order tensor. If there exists $x \in \mathbb{C}^{n} \backslash\{0\}$ such that

$$
\left\{\begin{array}{c}
A x^{m-1}=0 \\
x^{T} x=0
\end{array}\right.
$$

then $A$ is called irregular. Otherwise, $A$ is called regular $[15,17]$.

When $m$ is even, the E-characteristic polynomial $\psi_{A}(\lambda)$ is defined [15] as

$$
\psi_{A}(\lambda)=\operatorname{Res}_{x}\left(A x^{m-1}-\lambda\left(x^{T} x\right)^{\frac{m-2}{2}} x\right)=\sum_{j=0}^{h(m, n)} a_{j}(m, n) \lambda^{j},
$$

where the second equality is the expansion of the resultant of (1.2) in terms of $\lambda$, $h(m, n)$ is the highest power with generically non-zero coefficient, and $a_{j}(m, n)$ 's are polynomials in entries of $A$.

When $m$ is odd, the E-characteristic polynomial $\psi_{A}(\lambda)$ is defined [17] as

$$
\psi_{A}(\lambda)=\operatorname{Res}_{\left(x, x_{0}\right)}\left(\begin{array}{c}
A x^{m-1}-\lambda x_{0}^{m-2} x \\
x^{T} x-x_{0}^{2}
\end{array}\right)=\sum_{j=0}^{h(m, n)} a_{j}(m, n) \lambda^{2 j},
$$


where $2 h(m, n)$ is the highest power with generically non-zero coefficient.

Lemma 1.2. For a regular tensor, the E-characteristic polynomial can be defined as

$$
\operatorname{Res}_{\left(x, x_{0}\right)}\left(\begin{array}{c}
A x^{m-1}-\lambda x_{0}^{m-2} x \\
x^{T} x-x_{0}^{2}
\end{array}\right)
$$

for all $m$.

Proof. For a regular tensor and even $m$, the systems

$$
\left\{\begin{array}{c}
A x^{m-1}-\lambda\left(x_{0}\right)^{m-2} x=0 \\
x^{T} x-x_{0}^{2}=0
\end{array}\right.
$$

and

$$
A x^{m-1}-\lambda\left(x^{T} x\right)^{\frac{m-2}{2}} x=0
$$

are equivalent.

According to Theorem 4 of [17], any E-eigenvalue $\lambda$ of $A$ must be a root of $\psi_{A}(\lambda)$; if $A$ is regular, then a complex number $\lambda$ is an E-eigenvalue of $A$ if and only if it is a root of $\psi_{A}(\lambda)$. It was proved in [14] that when $m$ is even,

$$
h(m, n)=\frac{(m-1)^{n}-1}{m-2}=\sum_{i=0}^{n-1}(m-1)^{i} .
$$

Definition (1.1) is not a strict extension of the classical definition for eigenvalues of a square matrix, as it excludes complex eigenvalues whose eigenvectors satisfy $x^{T} x=0$ but $x \neq 0$ [17]. Recently, based upon this observation, Cartwright and Sturmfels [6] introduced equivalence classes of eigenpairs. If $\lambda \in \mathbb{C}$ and $x \in \mathbb{C}^{n} \backslash\{0\}$ satisfy

$$
A x^{m-1}=\lambda x,
$$

then $(\lambda, x)$ is called an eigenpair of $A$. Two eigenpairs $(\lambda, x)$ and $\left(\lambda^{\prime}, x^{\prime}\right)$ are considered to be equivalent if there is a complex number $t \neq 0$ such that $\lambda^{\prime}=t^{m-2} \lambda$ and $x^{\prime}=t x$. Cartwright and Sturmfels proved that (1.5) is true for all $m$ when counting the number of generalized eigenpairs without any normalization restriction [6].

An important property of a second-order tensor is that not only its eigenvalues but also the coefficients of its characteristic polynomial are invariants of that tensor. It was proved in $[15,17]$ that E-eigenvalues of a higher order tensor are invariants of that tensor. This implies that the coefficients of the E-characteristic polynomial, divided by the first nonzero coefficient, are invariants of that tensor; see Theorem 5 of [17]. There was no existing result about the invariance of the coefficients of the E-characteristic polynomial.

In [6], it was given that

$$
a_{0}(3,2)=\left(\operatorname{Res}_{x}\left(A x^{2}\right)\right)^{2},
$$

and mentioned in general that $a_{0}(m, n)$ is a power of $\operatorname{Res}_{x}\left(A x^{m-1}\right)$, without specifying the value of the power or giving a proof. When $m=3$ and $n=2$, they also present the coefficient of the leading term as the negative of the sum of two squares of linear combinations of the entries of $A$. 
In this paper, we explore more in this direction. The structure of the paper is as follows.

Since resultants are main tools to study the E-characteristic polynomials, we recall some facts about resultants in Section 2. In Section 3, we prove that all the coefficients of the E-characteristic polynomial are orthonormal invariants of a tensor. We then show that when $m$ is even, $a_{0}(m, n)$ is $\operatorname{Res}_{x}\left(A x^{m-1}\right)$, and when $m$ is odd, it is the square of $\operatorname{Res}_{x}\left(A x^{m-1}\right)$.

In Section 4, we give two simplified determinantal formulas for the E-characteristic polynomial when $n=2$. In the even case, this is a $(2 m-2) \times(2 m-2)$ determinant. In the odd case, this is a $(3 m-4) \times(3 m-4)$ determinant. The parameter $\lambda$ only appears in the first $m$ rows of the determinants.

We then address the leading coefficients of E-characteristic polynomials. Two sets of tensors are easy to handle: i) the set of tensors with infinitely many eigenpairs, and ii) the set of regular tensors. Actually, the E-characteristic polynomials of tensors in the first set are identically zero, while a complex number is an E-eigenvalue of a regular tensor if and only if it is a root of the E-characteristic polynomial of that tensor. For tensors with finitely many eigenpairs, we apply the perturbation method to approximate them by regular tensors with finitely many eigenpairs. Therefore we have to make sure that the perturbation is possible. Sections 5 and 6 serve this purpose. In Sections 5 and 6 , we prove that both the set of tensors with infinitely many eigenpairs and the set of irregular tensors have codimension at least 2 in the projective space of tensors. Therefore, the union of these two sets has codimension at least 2 in the projective space of tensors, which makes the approximation of a tensor on a hypersurface by regular tensors with finitely many eigenpairs possible.

Finally, in Section 7, we introduce the deficit system to explore the difference between E-eigenvalues and eigenpair equivalence classes of Cartwright and Sturmfels [6]. Based on this analysis, we show that when $n=2$, the leading coefficient of the E-characteristic polynomial is the $\left(\frac{m-2}{2}\right)$ th power of the sum of two squares when $m$ is even, and the negative of the $(m-2)$ th power of such a sum when $m$ is odd.

\section{Resultants}

We recall some results for resultants from [8] and [10].

For fixed positive integers $d_{1}, \cdots, d_{n}$, and each pair $(i, \alpha)$, where $i=1, \cdots n$ and $\alpha=\left(\alpha_{1}, \cdots, \alpha_{n}\right) \in \mathbb{Z}_{\geq 0}^{n}$ with $|\alpha|=\sum \alpha_{j}=d_{i}$, we introduce a variable $u_{i, \alpha}$. We have the following results about resultants:

Proposition 2.1. There is a unique polynomial $\operatorname{Res}_{x}=\operatorname{Res}_{d_{1}, \cdots, d_{n}} \in \mathbb{Z}\left[u_{i, \alpha}\right]$ with the following properties:

a. If $F_{1}, \cdots, F_{n} \in \mathbb{C}\left[x_{1}, \cdots, x_{n}\right]$ are homogeneous of degrees $d_{1}, \cdots, d_{n}$, then the equations $F_{1}=\cdots=F_{n}=0$ have a solution in $\mathbb{C P}^{n-1}$ if and only if $\operatorname{Res}\left(F_{1}, \cdots, F_{n}\right)=0$, where the notation $\operatorname{Res}\left(F_{1}, \cdots, F_{n}\right)$ means $\operatorname{Res}\left(c_{i, \alpha}\right)$, if

$$
F_{i}=\sum_{|\alpha|=d_{i}} c_{i, \alpha} x^{\alpha} .
$$

b. $\operatorname{Res}\left(x_{1}^{d_{1}}, \cdots, x_{n}^{d_{n}}\right)=1$.

c. Res is irreducible in $\mathbb{C}\left[u_{i, \alpha}\right]$.

Proposition 2.2. $\operatorname{Res}\left(F_{1}, \cdots, F_{n}\right)$ is a homogeneous polynomial in the coefficients of $F_{i}$, with degree $d_{1} \cdots d_{i-1} d_{i+1} \cdots d_{n}$, for $i=1, \cdots, n$. 
Proposition 2.3. If the $F_{j}$ 's are homogeneous of degree $d$, and $G_{i}=\sum a_{i j} F_{j}$, where $\left(a_{i j}\right)$ is an invertible matrix, then

$$
\operatorname{Res}\left(G_{1}, \cdots, G_{n}\right)=\operatorname{det}\left(a_{i j}\right)^{d^{n-1}} \operatorname{Res}\left(F_{1}, \cdots, F_{n}\right) .
$$

Proposition 2.4. If $F_{j}=F_{j}^{\prime} F_{j}^{\prime \prime}$ is a product of homogeneous polynomials, then

$$
\operatorname{Res}\left(F_{1}, \cdots, F_{j}, \cdots, F_{n}\right)=\operatorname{Res}\left(F_{1}, \cdots, F_{j}^{\prime}, \cdots, F_{n}\right) \operatorname{Res}\left(F_{1}, \cdots, F_{j}^{\prime \prime}, \cdots, F_{n}\right) .
$$

For an $n$-dimensional vector space $V$ (over $\mathbb{R}$ or $\mathbb{C}$ ), let $L: V \rightarrow V$ be a linear transformation. $L$ induces a natural map $L^{*}$ on functions on $V$. Fix a basis $\left\{e_{1}, \cdots, e_{n}\right\}$ of $V$, so that $x_{i}\left(\sum c_{j} e_{j}\right)=c_{i}$ defines a linear function $x_{i}$ on $V$, for $i=1, \cdots n$. If the transformation matrix of $L$ under this basis is $\left(a_{i j}\right)$, then $L^{*} x_{i}=\sum a_{i j} x_{j}$. So a polynomial $F$ in variables $x_{1}, \cdots x_{n}$ is a function on $V$, and $L^{*} F$ is a polynomial in the variables $x_{1}, \cdots x_{n}$ obtained from $F$ by substituting $x_{i}$ with $\sum a_{i j} x_{j}$.

Corollary 2.5. For an invertible linear transformation $L: V \rightarrow V$,

$$
\operatorname{Res}\left(L^{*} F_{1}, \cdots, L^{*} F_{n}\right)=\operatorname{det}\left(a_{i j}\right)^{d_{1} \cdots d_{n}} \operatorname{Res}\left(F_{1}, \cdots F_{n}\right) .
$$

Proof. Let

$$
F_{i}=\sum_{|\alpha|=d_{i}} u_{i, \alpha} x^{\alpha}
$$

Then $\operatorname{Res}\left(F_{1}, \cdots, F_{n}\right)$ is an irreducible polynomial in the variables $u_{i, \alpha}$, and by definition, if we denote

$$
L^{*} F_{i}=\sum_{|\alpha|=d_{i}} v_{i, \alpha} x^{\alpha},
$$

then $v_{i, \alpha}$ is a linear combination of $u$-variables $u_{j, \beta}$, and $\operatorname{Res}\left(L^{*} F_{1}, \cdots, L^{*} F_{n}\right)$ is obtained by substituting $u_{i, \alpha}$ for $v_{i, \alpha}$, so it is a polynomial in variables $u_{i, \alpha}$ and of the same degree as $\operatorname{Res}\left(F_{1}, \cdots, F_{n}\right)$.

Since the matrix $\left(a_{i j}\right)$ is invertible, the system $F_{1}=\cdots=F_{n}=0$ has a nontrivial solution if and only if the system $L^{*} F_{1}=\cdots=L^{*} F_{n}=0$ has a nontrivial solution, therefore $\operatorname{Res}\left(F_{1}, \cdots, F_{n}\right)$ is a divisor of $\operatorname{Res}\left(L^{*} F_{1}, \cdots, L^{*} F_{n}\right)$. We already know they have the same degree, so

$$
\operatorname{Res}\left(L^{*} F_{1}, \cdots, L^{*} F_{n}\right)=c \operatorname{Res}\left(F_{1}, \cdots, F_{n}\right),
$$

where $c$ is a constant.

We can determine this constant by checking the case $F_{i}=x_{i}^{d_{i}}, i=1, \cdots, n$. By definition,

$$
L^{*} x_{i}=\sum_{j} a_{i j} x_{j}
$$

Now, by Proposition 2.4 and the fact that $\operatorname{Res}_{1, \cdots, 1}$ is the standard determinant,

$$
\begin{aligned}
\operatorname{Res}\left(L^{*} F_{1}, \cdots, L^{*} F_{n}\right)= & \operatorname{Res}\left(\left(\sum a_{1 j} x_{j}\right)^{d_{1}}, \cdots,\left(\sum a_{n j} x_{j}\right)^{d_{n}}\right) \\
= & \operatorname{Res}\left(\sum a_{1 j} x_{j},\left(\sum a_{2 j} x_{j}\right)^{d_{2}}, \cdots,\left(\sum a_{n j} x_{j}\right)^{d_{n}}\right) \\
& \times \operatorname{Res}\left(\left(\sum a_{1 j} x_{j}\right)^{d_{1}-1}, \cdots,\left(\sum a_{n j} x_{j}\right)^{d_{n}}\right) \\
= & \cdots \\
= & \operatorname{Res}\left(\sum a_{1 j} x_{j},\left(\sum a_{2 j} x_{j}\right)^{d_{2}}, \cdots,\left(\sum a_{n j} x_{j}\right)^{d_{n}}\right)^{d_{1}} \\
= & \cdots \\
= & \operatorname{Res}\left(\sum a_{1 j} x_{j}, \sum a_{2 j} x_{j}, \cdots, \sum a_{n j} x_{j}\right)^{d_{1} \cdots d_{n}} \\
= & \operatorname{det}\left(a_{i j}\right)^{d_{1} \cdots d_{n}} .
\end{aligned}
$$


Hence,

$$
c=\operatorname{det}\left(a_{i j}\right)^{d_{1} \cdots d_{n}} .
$$

\section{Orthonormal invariance of the coefficients}

If $\psi_{A}(\lambda)$ is the E-characteristic polynomial (as defined in the introduction), let $a_{\bar{h}(m, n)}(m, n)$ be the first nonzero coefficient of $\psi_{A}(\lambda)$. Then, for $j=0, \cdots, \bar{h}(m, n)-1$, according to the relations between roots and coefficients of a one-variable polynomial,

$$
s_{\bar{h}(m, n)-j} \equiv(-1)^{\bar{h}(m, n)-j} a_{j}(m, n) / a_{\bar{h}(m, n)}(m, n)
$$

is the sum of all possible products of $\bar{h}(m, n)-j$ E-eigenvalues, hence an elementary symmetric function of the E-eigenvalues.

In the following discussion, we distinguish between a tensor $\mathcal{A}$ and its hypermatrix $A$ in a coordinate system.

Let $V$ be an n-dimensional vector space over $\mathbb{R}$ with a Euclidean inner product $<,>$. Let $\mathcal{A}$ be an $m$ th order covariant tensor on $V$, that is,

$$
\mathcal{A}: V^{m} \rightarrow \mathbb{R}
$$

is a multi-linear function. In particular, a first order covariant tensor is called a covariant vector, denoted by $\mathbf{x}$. In practice, what we consider are complexified tensors, i.e., we view them as multiple-linear functions from $V^{m} \otimes_{\mathbb{R}} \mathbb{C} \rightarrow \mathbb{C}$.

Choose an orthonormal frame $E=\left\{e_{1}, \ldots, e_{n}\right\}$ in $V$, and define

$$
\mathcal{A}\left(e_{i_{1}}, \ldots, e_{i_{m}}\right):=a_{i_{1} i_{2} \cdots i_{m}}, \mathbf{x}\left(e_{i}\right)=x_{i} .
$$

Then $\left(a_{i_{1} i_{2} \cdots i_{m}}\right)$ (resp. $x_{i}$ ) is the hypermatrix of $\mathcal{A}$ (resp. $\mathbf{x}$ ) with respect to the frame $E$. If all $a_{i_{1} i_{2} \cdots i_{m}}$, for $i_{1}, \cdots, i_{m}=1, \cdots, n$, are real, then we say that $\mathcal{A}$ is real. If all $x_{i}$, for $i=1, \cdots, n$, are real, then we say that $\mathbf{x}$ is real.

If we choose another orthonormal frame $\tilde{E}=\left\{\tilde{e}_{1}, \ldots, \tilde{e}_{n}\right\}$, that is,

$$
\tilde{e}_{i}=\sum C_{i}^{j} e_{j}
$$

where the matrix $\left(C_{i}^{j}\right) \in O(n)$ is the transformation matrix, then the hypermatrices of $\mathcal{A}$ and $\mathrm{x}$ with respect to $\tilde{e}_{1}, \ldots, \tilde{e}_{n}$ are given by

$$
\begin{gathered}
\tilde{a}_{i_{1} i_{2} \cdots i_{m}}=\sum C_{i_{1}}^{j_{1}} \ldots C_{i_{m}}^{j_{m}} a_{j_{1} j_{2} \cdots j_{m}}, \\
\tilde{x}_{i}=\sum C_{i}^{j} x_{j} .
\end{gathered}
$$

REMARK 3.1. For a tensor, the hypermatrices with respect to different orthonormal frames are orthogonally similar.

REMARK 3.2. Since we only consider tensors under orthonormal transformations, we do not distinguish between covariant tensors and contravariant tensors.

For a complex $m$ th order covariant tensor $\mathcal{A}$ and a covariant vector $\mathbf{x}$, we can define a covariant vector $\mathcal{A} \mathrm{x}^{m-1}$ by

$$
\mathcal{A} \mathbf{x}^{m-1}\left(e_{i}\right)=\sum_{i_{2}, \cdots, i_{m}=1}^{n} \mathcal{A}\left(e_{i}, e_{i_{2}}, \cdots, e_{i_{m}}\right) \mathbf{x}\left(e_{i_{2}}\right) \cdots \mathbf{x}\left(e_{i_{m}}\right),
$$


for $i=1, \cdots, n$, and any orthonormal frame $E=\left\{e_{1}, \ldots, e_{n}\right\}$ in $V$. For any covariant vector $\mathbf{x}$, define

$$
\mathbf{x}^{T} \mathbf{x}=\sum_{i=1}^{n}\left(\mathbf{x}\left(e_{i}\right)\right)^{2}
$$

for any orthonormal frame $E=\left\{e_{1}, \ldots, e_{n}\right\}$ in $V$. By (3.1) and (3.2), we see that the definitions (3.3) and (3.4) are well-defined, independent of the frame $E$.

For a complex $m$ th order covariant tensor $\mathcal{A}$, if $\lambda \in \mathbb{C}$ and the non-zero covariant vector $\mathbf{x}$ satisfy

$$
\left\{\begin{array}{c}
\mathcal{A} \mathbf{x}^{m-1}=\lambda \mathbf{x} \\
\mathbf{x}^{T} \mathbf{x}=1
\end{array}\right.
$$

then $\lambda$ is an E-eigenvalue of $\mathcal{A}$ and $\mathbf{x}$ is an E-eigenvector of $\mathcal{A}$. By the above discussion, we see that $\lambda$ is invariant, i.e., it is independent of the choice of the frame.

We say that a fact or quantity is an orthonormal invariant if it is invariant under changes of orthonormal frames.

Fix an orthonormal frame $E=\left\{e_{1}, \ldots, e_{n}\right\}$ in $V$, let the hypermatrix $A=\left(a_{i_{1} i_{2} \cdots i_{m}}\right)$ (resp. $x_{i}$ ) be the hypermatrix of $\mathcal{A}($ resp. x), and let

$$
F_{i}(x)=\sum_{i_{2}, \cdots, i_{m}=1}^{n} a_{i i_{2} \cdots i_{m}} x_{i_{2}} \cdots x_{i_{m}} .
$$

We use the notation $\operatorname{Res}_{\mathbf{x}}\left(\mathcal{A} \mathbf{x}^{m-1}\right)$ to denote $\operatorname{Res}_{x}\left(F_{1}, \ldots, F_{n}\right)$, and $\psi_{\mathcal{A}}(\lambda)$ to denote $\psi_{A}(\lambda)$.

TheOREm 3.3. $\operatorname{Res}_{\mathbf{x}}\left(\mathcal{A} \mathbf{x}^{m-1}\right)$ is an orthonormal invariant of $\mathcal{A}$. Furthermore, all the coefficients of $\psi_{\mathcal{A}}(\lambda)$ are orthonormal invariants of $\mathcal{A}$.

Proof. We first prove that $\operatorname{Res}_{\mathbf{x}}\left(\mathcal{A} \mathbf{x}^{m-1}\right)$ is an orthonormal invariant of $\mathcal{A}$.

If we choose another orthonormal frame $\tilde{e}_{1}, \ldots, \tilde{e}_{n}$,

$$
\tilde{e}_{i}=\sum C_{i}^{j} e_{j}
$$

and denote

$$
\tilde{F}_{i}(\tilde{x})=\sum_{i_{2}, \cdots, i_{m}=1}^{n} \tilde{a}_{i i_{2} \cdots i_{m}} \tilde{x}_{i_{2}} \cdots \tilde{x}_{i_{m}},
$$

then by a direct calculation we get

$$
\tilde{F}_{i}(\tilde{x})=\sum C_{i}^{j} F_{j}(x) .
$$

So if we let

$$
G_{i}(x)=\sum C_{i}^{j} F_{j}(x)
$$

then by Proposition 2.3,

$$
\operatorname{Res}_{x}\left(G_{1}, \ldots, G_{n}\right)=\operatorname{det}\left(C_{i}^{j}\right)^{(m-1)^{n-1}} \operatorname{Res}_{x}\left(F_{1}, \ldots, F_{n}\right) .
$$


Since

$$
\tilde{F}_{i}(\tilde{x})=G_{i}\left(\sum_{j} C_{j}^{1} \tilde{x}_{j}, \cdots, \sum_{j} C_{j}^{n} \tilde{x}_{j}\right),
$$

by the corollary of Proposition 2.4 we have

$$
\operatorname{Res} \tilde{x}\left(\tilde{F}_{1}, \ldots, \tilde{F}_{n}\right)=\operatorname{det}\left(C_{i}^{j}\right)^{(m-1)^{n}} \operatorname{Res}_{x}\left(G_{1}, \ldots, G_{n}\right) .
$$

Since $\operatorname{det}\left(C_{i}^{j}\right)= \pm 1,(m-1)^{n-1} m$ is even, we have

$$
\operatorname{Res}_{\tilde{x}}\left(\tilde{F}_{1}, \ldots, \tilde{F}_{n}\right)=\operatorname{Res}_{x}\left(F_{1}, \ldots, F_{n}\right) .
$$

By the same method we can prove that when $m$ is even, $\operatorname{Res}_{\mathbf{x}}\left(\mathcal{A} \mathbf{x}^{m-1}\right.$ $\left.-\lambda\left(\mathbf{x}^{T} \mathbf{x}\right)^{\frac{m-2}{2}} \mathbf{x}\right)$ is an orthonormal invariant, and when $m$ is odd, $\operatorname{Res}_{\left(\mathbf{x}, x_{0}\right)}\left(\begin{array}{c}\mathcal{A} \mathbf{x}^{m-1}-\lambda x_{0}^{m-2} \mathbf{x} \\ \mathbf{x}^{T} \mathbf{x}-x_{0}^{2}\end{array}\right)$ is orthonormal invariant.

Because of this theorem, we need not strictly distinguish between a tensor and its corresponding hypermatrix in further discussion.

Lemma 3.4. For a hypermatrix $A, \operatorname{Res}_{x}\left(A x^{m-1}\right)$ is an irreducible polynomial in the entries of $A$ (viewed as variables).

Proof. Any index $\alpha=\left(\alpha_{1}, \cdots, \alpha_{n}\right)$ with $|\alpha|=m-1$ defines a subset $S_{\alpha}$ of $\{1, \cdots, n\}^{m-1}$ :

$S_{\alpha}=\left\{\left(i_{2}, \cdots, i_{m}\right) \mid\right.$ among $i_{2}, \cdots, i_{m}, \alpha_{j}$ of them are equal to $j$, for $\left.j=1, \cdots, n\right\}$.

Obviously,

$$
\begin{gathered}
S_{\alpha} \cap S_{\beta}=\phi, \text { for any } \alpha \neq \beta, \\
\cup_{\alpha} S_{\alpha}=\{1, \cdots, n\}^{m-1} .
\end{gathered}
$$

For the system $A x^{m-1}=0$, if we denote the equations by

$$
F_{i}=\sum c_{i \alpha} x^{\alpha}
$$

$i=1, \cdots, n$, then

$$
c_{i \alpha}=\sum_{\left(i_{2}, \cdots, i_{m}\right) \in S_{\alpha}} a_{i i_{2}, \cdots, i_{m}} .
$$

Assume $\operatorname{Res}_{x}\left(A x^{m-1}\right)=P\left(c_{i \alpha}\right)=f\left(a_{i_{1} \cdots i_{m}}\right) g\left(a_{i_{1} \cdots i_{m}}\right)$. Let us fix a function

$$
\phi:\left\{\alpha\left|\alpha=\left(\alpha_{1}, \cdots, \alpha_{n}\right),\right| \alpha \mid=m-1\right\} \rightarrow\{1, \cdots, n\}^{m-1}
$$

such that $\phi(\alpha) \in S_{\alpha}$. We can choose entries of $A$ such that for every $c_{i \alpha}$ the only nonzero entry is $a_{i \phi(\alpha)}$. Then $\operatorname{Res}_{x}\left(A x^{m-1}\right)$ is an irreducible polynomial in the variables corresponding to these nonzero entries. Hence,

$$
P\left(a_{i \phi(\alpha)}\right)=f\left(a_{i \phi(\alpha)}\right) g\left(a_{i \phi(\alpha)}\right)
$$


Since $P$ is irreducible, one of $f\left(a_{i \phi(\alpha)}\right)$ or $g\left(a_{i \phi(\alpha)}\right)$, say $g\left(a_{i \phi(\alpha)}\right)$, has to be of degree 0 and the degree of the other is equal to the degree of $P$. Then $g\left(a_{i_{1} \cdots i_{m}}\right)$ has degree 0 because $P\left(a_{i_{1} \cdots i_{m}}\right)$ has the same degree as $P\left(c_{i \alpha}\right)$. Therefore $\operatorname{Res}_{x}\left(A x^{m-1}\right)$ is irreducible.

We now give a general formula for $a_{0}(m, n)$.

Theorem 3.5. When $m$ is even, we have

$$
a_{0}(m, n)=\operatorname{Res}_{x}\left(A x^{m-1}\right) .
$$

When $m$ is odd, we have

$$
a_{0}(m, n)=\left(\operatorname{Res}_{x}\left(A x^{m-1}\right)\right)^{2} .
$$

Proof. When $m$ is even, by (1.3) we have

$$
a_{0}(m, n)=\psi_{A}(0)=\operatorname{Res}_{x}\left(A x^{m-1}\right) .
$$

This proves (3.5).

When $m$ is odd, by (1.4) we have

$$
a_{0}(m, n)=\psi_{A}(0)=\operatorname{Res}_{\left(x, x_{0}\right)}\left(\begin{array}{c}
A x^{m-1} \\
x^{T} x-x_{0}^{2}
\end{array}\right) .
$$

To prove (3.6), it suffices now to prove

$$
\operatorname{Res}_{\left(x, x_{0}\right)}\left(\begin{array}{c}
A x^{m-1} \\
x^{T} x-x_{0}^{2}
\end{array}\right)=\left(\operatorname{Res}_{x}\left(A x^{m-1}\right)\right)^{2} .
$$

We first show that $\left(\operatorname{Res}_{x}\left(A x^{m-1}\right)\right)^{2}$ has the correct degree. Denote $\left(A x^{m-1}\right)_{i}$ by $F_{i}(x)$ for $i=1 \cdots n$. Since $x^{T} x-x_{0}^{2}$ has degree 2 , by Proposition $2.2 \operatorname{Res}_{\left(x, x_{0}\right)}\left(\begin{array}{c}A x^{m-1} \\ x^{T} x-x_{0}^{2}\end{array}\right)$ is a homogeneous polynomial in the coefficients of $F_{i}(x)$, for any $i$, with degree $2(m-$ $1)^{n-1}$. Obviously, for any $i,\left(\operatorname{Res}_{x}\left(A x^{m-1}\right)\right)^{2}$ is a homogeneous polynomial in the coefficients of $F_{i}(x)$, with degree $2(m-1)^{n-1}$. This shows that $\left(\operatorname{Res}_{x}\left(A x^{m-1}\right)\right)^{2}$ has the correct degree.

Next we prove that the system

$$
\left\{\begin{array}{c}
A x^{m-1}=0 \\
x^{T} x=x_{0}^{2}
\end{array}\right.
$$

has a nonzero solution if and only if $\operatorname{Res}_{x}\left(A x^{m-1}\right)=0$. Let $\left(x, x_{0}\right)$ be a nonzero solution of (3.8). Since $A$ is regular, $x_{0} \neq 0$. By the last equation of (3.8), $x \neq 0$. Then $x$ is a nonzero solution of

$$
A x^{m-1}=0 .
$$

Thus, $\operatorname{Res}_{x}\left(A x^{m-1}\right)=0$. On the other hand, suppose that $\operatorname{Res}_{x}\left(A x^{m-1}\right)=0$. Then (3.9) has a nonzero solution $x$. Let $x_{0}^{2}=x^{T} x$. Then $\left(x, x_{0}\right)$ is a nonzero solution 
of (3.8). Therefore $\operatorname{Res}_{\left(x, x_{0}\right)}\left(\begin{array}{c}A x^{m-1} \\ x^{T} x-x_{0}^{2}\end{array}\right)=0$ and $\operatorname{Res}_{x}\left(A x^{m-1}\right)=0$ define the same varieties. By the irreducibility of $\operatorname{Res}_{x}\left(A x^{m-1}\right)$, we know

$$
\operatorname{Res}_{\left(x, x_{0}\right)}\left(\begin{array}{c}
A x^{m-1} \\
x^{T} x-x_{0}^{2}
\end{array}\right)=\left(\operatorname{Res}_{x}\left(A x^{m-1}\right)\right)^{k} .
$$

Then by degree, we know $k=2$. This proves (3.7).

This shows the importance of $\operatorname{Res}_{x}\left(A x^{m-1}\right)$. When $m=2$, it is the determinant of the square matrix $A$ in the classical sense. Hence, it is a genuine extension of the determinant of a square matrix, and deserves to be studied further.

Before ending this section, we give a proposition on the degree of the coefficients of $\psi_{A}(\lambda)$ as polynomials in the entries of $A$.

When $m$ is even, we may see that

$$
A x^{m-1}-\lambda\left(x^{T} x\right)^{\frac{m-2}{2}} x=0
$$

is a system of homogeneous polynomials in $x$. Every equation of (3.10) has the same degree $m-1$. Thus, by Proposition $2.2, \psi_{A}(\lambda)$ is a homogeneous polynomial in the entries of $A$ and $\lambda$, with degree $n(m-1)^{n-1}$. Hence, in (1.3), $a_{j}(m, n)$ is a homogeneous polynomial in the entries of $A$, with degree $n(m-1)^{n-1}-j$. When $m$ is odd, the first $m$ equations of

$$
\left\{\begin{array}{c}
A x^{m-1}=\lambda x_{0}^{m-2} x \\
x^{T} x=x_{0}^{2}
\end{array}\right.
$$

have the same degree $m-1$, while the coefficients of last equation of (3.11) are either 1 or -1 . Thus, by Proposition 2.2, $\psi_{A}(\lambda)$ is a homogeneous polynomial in the entries of $A$ and $\lambda$, with degree $2 n(m-1)^{n-1}$. Hence, in (1.4), $a_{j}(m, n)$ is a homogeneous polynomial in the entries of $A$, with degree $2 n(m-1)^{n-1}-2 j$. We now have the following proposition.

Proposition 3.6. In (1.3), $a_{j}(m, n)$ is a homogeneous polynomial in the entries of $A$, with degree $n(m-1)^{n-1}-j$. In particular, $a_{h(m, n)}(m, n)$ is a homogeneous polynomial in the entries of $A$, with degree $n(m-1)^{n-1}-\frac{(m-1)^{n}-1}{m-2}$.

In (1.4), $a_{j}(m, n)$ is a homogeneous polynomial in the entries of $A$, with degree $2 n(m-1)^{n-1}-2 j$. In particular, $a_{h(m, n)}(m, n)$ is a homogeneous polynomial in the entries of $A$, with degree $2 n(m-1)^{n-1}-\frac{2(m-1)^{n}-2}{m-2}$.

\section{E-characteristic polynomials when $n=2$}

We now derive some simplified forms for $\psi_{\mathcal{A}}(\lambda)$ in the case that $n=2$.

Let $b_{j}=\sum\left\{a_{1 i_{2} \cdots i_{m}}\right.$ : exactly $j-1$ of $i_{2}, \cdots, i_{m}$ are 2$\}$ for $j=1, \cdots, m$, and $c_{j}=$ $\sum\left\{a_{2 i_{2} \cdots i_{m}}\right.$ : exactly $j-1$ of $i_{2}, \cdots, i_{m}$ are 2$\}$ for $j=1, \cdots, m$. For $j=1, \cdots, m-1$, let $d_{j}=b_{j}-c_{j+1}$, and let $d_{m}=b_{m}$.

For an $N \times N$ matrix $M=\left(m_{i j}\right)$, just like $\left\{m_{11}, m_{22}, \cdots, m_{N N}\right\}$ is its diagonal, we call $\left\{m_{1,2 k+1}, m_{2,2 k+2}, \cdots, m_{N-2 k, N}\right\}$ the $k$ th even upper sub-diagonal of $M$ for positive $k$ such that $2 k+1 \leq N$.

We now discuss the case that $m$ is even.

TheOREM 4.1. Suppose that $m=2 k+2$ and $n=2$, where $k \geq 0$. Let

$$
\bar{b}_{2 j+1}=b_{2 j+1}-\left(\begin{array}{c}
k \\
j
\end{array}\right) \lambda \text { and } \bar{c}_{2 j+2}=c_{2 j+2}-\left(\begin{array}{c}
k \\
j
\end{array}\right) \lambda,
$$


for $j=0, \cdots, k$. Then for a regular $A, \psi_{A}(\lambda)$ is the determinant of the following $(2 m-2) \times(2 m-2)$ matrix:

$$
M_{1}=\left(\begin{array}{cccccccccc}
\bar{b}_{1} & b_{2} & \bar{b}_{3} & \cdots & \bar{b}_{m-1} & b_{m} & 0 & 0 & \cdots & 0 \\
0 & \bar{b}_{1} & b_{2} & \cdots & b_{m-2} & \bar{b}_{m-1} & b_{m} & 0 & \cdots & 0 \\
\cdot & \cdot & \cdot & \cdots & \cdot & \cdot & \cdot & \cdot & \cdots & \cdot \\
\vdots & \vdots & \vdots & \ddots & \vdots & \vdots & \vdots & \vdots & \ddots & \vdots \\
0 & 0 & 0 & \cdots & \bar{b}_{1} & b_{2} & \bar{b}_{3} & b_{4} & \cdots & b_{m} \\
0 & 0 & 0 & \cdots & c_{1} & \bar{c}_{2} & c_{3} & \bar{c}_{4} & \cdots & \bar{c}_{m} \\
-c_{1} & d_{1} & d_{2} & \cdots & d_{m-2} & d_{m-1} & b_{m} & 0 & \cdots & 0 \\
0 & -c_{1} & d_{1} & \cdots & d_{m-3} & d_{m-2} & d_{m-1} & b_{m} & \cdots & 0 \\
\vdots & \vdots & \vdots & \ddots & \vdots & \vdots & \vdots & \vdots & \ddots & \vdots \\
0 & 0 & 0 & \cdots & d_{1} & d_{2} & d_{3} & d_{4} & \cdots & b_{m}
\end{array}\right) .
$$

Here, the first $m-1$ entries of the diagonal and the first $\frac{m-2}{2}$ even upper sub-diagonals of $M_{1}$ are $\bar{b}_{1}, \bar{b}_{3}, \cdots, \bar{b}_{m-1}$, while the $m$ th entries of the diagonal and the first $\frac{m-2}{2}$ even upper sub-diagonals of $M_{1}$ are $\bar{c}_{2}, \bar{c}_{4}, \cdots, \bar{c}_{m}$. They are linear factors of $\lambda$.

Proof. Consider

$$
F(x, \lambda) \equiv\left(\begin{array}{c}
\left(A x^{m-1}\right)_{1}-\lambda\left(x^{T} x\right)^{\frac{m-2}{2}} x_{1} \\
x_{2}\left(A x^{m-1}\right)_{1}-x_{1}\left(A x^{m-1}\right)_{2}
\end{array}\right)=0 .
$$

Since $A$ is regular, any nonzero solution of (3.10) is a nonzero solution of (4.2). This implies that $\psi_{A}(\lambda)$ is a factor of $\operatorname{Res}_{x} F(x, \lambda)$. On the other hand, the only possible additional nonzero solution of (4.2) satisfies $x_{1}=0$ and $\left(A x^{m-1}\right)_{1}=0$. Since $\left(A x^{m-1}\right)_{1}=$ $\sum_{i=1}^{m} b_{i} x_{1}^{m-i} x_{2}^{i-1}, x_{1}=0, x_{2} \neq 0$, and $\left(A x^{m-1}\right)_{1}=0$ imply that $b_{m}=a_{12 \cdots 2}=0$. Therefore, we conclude that

$$
\operatorname{Res}_{x} F(x, \lambda)=b_{m} \psi_{A}(\lambda)
$$

Now, we have

$$
F(x, \lambda)=\left(\begin{array}{c}
\sum_{j=0}^{k}\left[\bar{b}_{2 j+1} x_{1}^{m-2 j-1} x_{2}^{2 j}+b_{2 j+2} x_{1}^{m-2 j-2} x_{2}^{2 j+1}\right] \\
-c_{1} x_{1}^{m}+\sum_{i=1}^{m-1} d_{i} x_{1}^{m-i} x_{2}^{i}+d_{m} x_{2}^{m}
\end{array}\right)
$$

By the Sylvester formula $[8,10], \operatorname{Res}_{x} F(x, \lambda)$ is the determinant of the following $(2 m-1) \times(2 m-1)$ matrix:

$$
\left(\begin{array}{ccccccccccc}
\bar{b}_{1} & b_{2} & \bar{b}_{3} & \cdots & \bar{b}_{m-1} & b_{m} & 0 & 0 & \cdots & 0 & 0 \\
0 & \bar{b}_{1} & b_{2} & \cdots & b_{m-2} & \bar{b}_{m-1} & b_{m} & 0 & \cdots & 0 & 0 \\
\cdot & \cdot & \cdot & \cdots & \cdot & \cdot & \cdot & \cdot & \cdots & \cdot & \cdot \\
\vdots & \vdots & \vdots & \ddots & \vdots & \vdots & \vdots & \vdots & \ddots & \vdots & \vdots \\
0 & 0 & 0 & \cdots & \bar{b}_{1} & b_{2} & \bar{b}_{3} & b_{4} & \cdots & b_{m} & 0 \\
0 & 0 & 0 & \cdots & 0 & \bar{b}_{1} & b_{2} & \bar{b}_{3} & \cdots & \bar{b}_{m-1} & b_{m} \\
-c_{1} & d_{1} & d_{2} & \cdots & d_{m-2} & d_{m-1} & b_{m} & 0 & \cdots & 0 & 0 \\
0 & -c_{1} & d_{1} & \cdots & d_{m-3} & d_{m-2} & d_{m-1} & b_{m} & \cdots & 0 & 0 \\
\vdots & \vdots & \vdots & \ddots & \vdots & \vdots & \vdots & \vdots & \ddots & \vdots & \vdots \\
0 & 0 & 0 & \cdots & d_{1} & d_{2} & d_{3} & d_{4} & \cdots & b_{m} & 0 \\
0 & 0 & 0 & \cdots & -c_{1} & d_{1} & d_{2} & d_{3} & \cdots & d_{m-1} & b_{m}
\end{array}\right) .
$$


As elementary row and column operations preserve the value of the determinant, we may subtract the last row from the $m$ th row in the above matrix. Then all the elements of the last column of the matrix are zero except the bottom element, which is $b_{m}$. Then we may delete the last column and the last row, and extract $b_{m}$ from the determinant. Then we see that $\operatorname{Res}_{x} F(x, \lambda)=b_{m} \operatorname{det}\left(M_{1}\right)$. Comparing with (4.3), we have $\psi_{A}(\lambda)=\operatorname{det}\left(M_{1}\right)$. The proof is complete.

The merit of $M_{1}$ is that only the first $m$ entries of the diagonal and the first $\frac{m-2}{2}$ even upper sub-diagonals of $M_{1}$ contain linear factors of $\lambda$, i.e., those $\bar{b}_{i}$ and $\bar{c}_{i}$. By some elementary column operations, we may eliminate $\lambda$ in the terms other than $\bar{b}_{1}$ and $\bar{c}_{2}$.

When $m=4$ and $n=2$, we have $h(4,2)=4$, and

$$
\begin{aligned}
& a_{h(4,2)=4}(4,2)=\left(b_{1}-c_{2}-b_{3}+c_{4}\right)^{2}+\left(c_{1}+b_{2}-c_{3}-b_{4}\right)^{2} \\
= & \left(a_{1111}+a_{2222}-a_{1221}-a_{1212}-a_{1122}-a_{2211}-a_{2121}-a_{2112}\right)^{2} \\
& +\left(a_{1211}+a_{1121}+a_{1112}+a_{2111}-a_{1222}-a_{2221}-a_{2212}-a_{2122}\right)^{2} .
\end{aligned}
$$

We now discuss the case where $m$ is odd. Let $e_{1}=b_{1} c_{1}, e_{2}=b_{1} c_{2}+$ $b_{2} c_{1}, e_{3}=b_{1} c_{3}+b_{2} c_{2}+b_{3} c_{1}, \cdots, e_{m}=b_{1} c_{m}+b_{2} c_{m-1}+\cdots+b_{m} c_{1}, e_{m+1}=b_{2} c_{m}+\cdots+$ $b_{m} c_{2}, \cdots, e_{2 m-1}=b_{m} c_{m}$.

Theorem 4.2. Suppose that $m=k+2$ and $n=2$, where $k \geq 1$ is odd. Let

$$
\bar{e}_{2 j}=e_{2 j}-\left(\begin{array}{c}
k \\
j
\end{array}\right) \lambda^{2}
$$

for $j=1, \cdots, m-1$. Let $\bar{f}_{i}=\bar{e}_{i}+b_{1} d_{i+1}$ for $i=2,4, \cdots, m-1 ; f_{i}=e_{i}+b_{1} d_{i+1}$ for $i=$ $3,5, \cdots, m-2 ; f_{i}=e_{i}-c_{m} d_{i-m+1}$ for $i=m, m+2, \cdots, 2 m-3 ; \bar{f}_{i}=\bar{e}_{i}-c_{m} d_{i-m+1}$ for $i=m+1, m+3, \cdots, 2 m-2$. Then $\psi_{A}(\lambda)$ is the determinant of the following (3m$4) \times(3 m-4)$ matrix:

$$
M_{2}=\left(\begin{array}{cccccccccc}
\bar{f}_{2} & f_{3} & \cdots & \bar{f}_{m-1} & e_{m} & \cdots & \bar{e}_{2 m-2} & e_{2 m-1} & \cdots & 0 \\
e_{1} & \bar{e}_{2} & \cdots & e_{m-2} & \bar{e}_{m-1} & \cdots & e_{2 m-3} & \bar{e}_{2 m-2} & \cdots & 0 \\
\vdots & \vdots & \ddots & \vdots & \vdots & \ddots & \vdots & \vdots & \ddots & \vdots \\
0 & 0 & \cdots & \bar{e}_{2} & e_{3} & \cdots & \bar{f}_{m-1} & f_{m} & \cdots & \bar{f}_{2 m-2} \\
-c_{1} & d_{1} & \cdots & d_{m-1} & b_{m} & \cdots & 0 & 0 & \cdots & 0 \\
0 & -c_{1} & \cdots & d_{m-2} & d_{m-1} & \cdots & 0 & 0 & \cdots & 0 \\
\vdots & \vdots & \ddots & \vdots & \vdots & \ddots & \vdots & \vdots & \ddots & \vdots \\
. & . & \cdots & . & . & \cdots & . & . & \cdots & . \\
0 & 0 & \cdots & 0 & 0 & \cdots & d_{2} & d_{3} & \cdots & 0 \\
0 & 0 & \cdots & 0 & 0 & \cdots & d_{1} & d_{2} & \cdots & b_{m}
\end{array}\right) .
$$

Here, the first $m-2$ entries of the first row and the last $m-2$ entries of the $m$ th row are somewhat different from the other entries of the first $m$ rows, with $e_{i}$ or $\bar{e}_{i}$ being replaced by $f_{i}$ or $\bar{f}_{i}$.

Proof. Consider

$$
G(x, \lambda) \equiv\left(\begin{array}{c}
\left(A x^{m-1}\right)_{1}\left(A x^{m-1}\right)_{2}-\lambda^{2}\left(x^{T} x\right)^{\frac{2 m-4}{2}} x_{1} x_{2} \\
x_{2}\left(A x^{m-1}\right)_{1}-x_{1}\left(A x^{m-1}\right)_{2}
\end{array}\right)=0 .
$$


Similar to the proof of Theorem 4.1, we may conclude that

$$
\operatorname{Res}_{x} G(x, \lambda)=b_{m} c_{1} \psi_{A}(\lambda) \text {. }
$$

Now, we have

$$
G(x, \lambda)=\left(\begin{array}{c}
\sum_{j=0}^{m-1} e_{2 j+1} x_{1}^{2 m-2 j-2} x_{2}^{2 j}+\sum_{j=1}^{m-1} \bar{e}_{2 j} x_{1}^{2 m-2 j-1} x_{2}^{2 j-1} \\
-c_{1} x_{1}^{m}+\sum_{i=1}^{m-1} d_{i} x_{1}^{m-i} x_{2}^{i}+d_{m} x_{2}^{m}
\end{array}\right) .
$$

By the Sylvester formula $[8,10], \operatorname{Res}_{x} G(x, \lambda)$ is the determinant of the following $(3 m-2) \times(3 m-2)$ matrix:

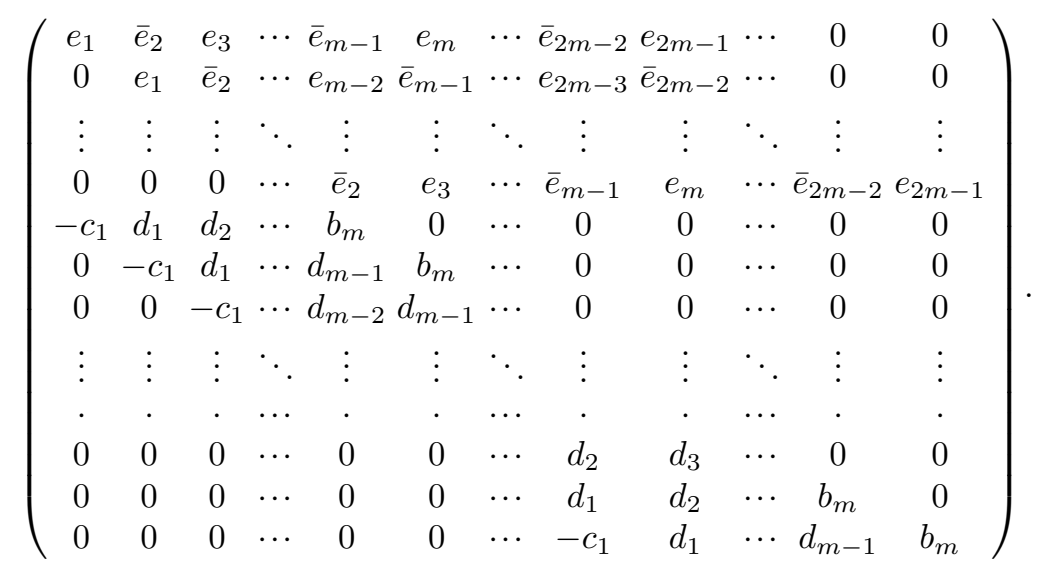

Note that $e_{1}=b_{1} c_{1}$ and $e_{2 m-1}=b_{m} c_{m}$. Thus, as elementary row and column operations preserve the value of the determinant, we may eliminate $e_{1}$ in the first column with a multiple of the $(\mathrm{m}+1)$ th row, and eliminate $e_{2 m-1}$ in the last column with a multiple of the last row. Then we may delete the first column, the last column, the $(m+1)$ th row and the last row, and extract $b_{m} c_{1}$ from the determinant. We see that $\operatorname{Res}_{x} G(x, \lambda)=b_{m} c_{1} \operatorname{det}\left(M_{2}\right)$. Comparing with (4.6), we have $\psi_{A}(\lambda)=\operatorname{det}\left(M_{2}\right)$. The proof is complete.

The merit of $M_{2}$ is that only the first $m$ entries of the diagonal and the first $m-2$ even upper sub-diagonals of $M_{2}$ contain linear factors of $\lambda^{2}$, i.e., those $\bar{e}_{i}$ and $\bar{f}_{i}$. By some elementary column operations, we may eliminate $\lambda$ in the terms other than $\bar{f}_{2}$ and $\bar{e}_{2}$.

When $m=3$ and $n=2$, in [6], it was given that

$$
a_{h(3,2)=3}(3,2)=-\left(-a_{111}+a_{122}+a_{212}+a_{221}\right)^{2}-\left(a_{112}+a_{121}+a_{211}-a_{222}\right)^{2} .
$$

If we use $b_{i}$ and $c_{i}$ to write this, then we have

$$
a_{h(3,2)=3}(3,2)=-\left(b_{1}-c_{2}-b_{3}\right)^{2}-\left(c_{1}+b_{2}-c_{3}\right)^{2} .
$$

We see that in the form using $b_{i}$ and $c_{i}, a_{h(3,2)}(3,2)$ and $a_{h(4,2)}(4,2)$ are very similar. Let $P_{m}$ and $Q_{m}$ be the sum of the first $m$ terms of the following two series respectively:

$$
b_{1}-c_{2}-b_{3}+c_{4}+b_{5}-c_{6}-b_{7}+c_{8}+b_{9}-c_{10}-\cdots
$$

and

$$
c_{1}+b_{2}-c_{3}-b_{4}+c_{5}+b_{6}-c_{7}-b_{8}+c_{9}+b_{10}-\cdots,
$$


where the signs of these terms are cyclically changed with the cycle four. Then, we may write

$$
a_{h(3,2)}(3,2)=-\left(P_{3}^{2}+Q_{3}^{2}\right)
$$

and

$$
a_{h(4,2)}(4,2)=P_{4}^{2}+Q_{4}^{2} .
$$

Using Theorem 4.1, we find via computation that

$$
a_{h(6,2)}(6,2)=\left(P_{6}^{2}+Q_{6}^{2}\right)^{2} .
$$

It seems that there are formulas for $a_{h(m, 2)}(m, 2)$ via $P_{m}^{2}+Q_{m}^{2}$ for all $m \geq 2$. Later we will show that this is true.

\section{A bound on the codimension of the variety of tensors with infinitely many eigenpairs}

For positive integers $d_{1}, d_{2}, \cdots, d_{n}$, let $\mathbb{P}$ be the complex projective space corresponding to the homogeneous coordinate ring

$$
\mathbb{C}\left[u_{i \alpha}|| \alpha \mid=\alpha_{0}+\cdots+\alpha_{n}=d_{i}, i=1, \cdots n\right] .
$$

Now for a system of homogeneous polynomial equations: $F_{1}=F_{2}=\cdots=F_{n}=0$ where $F_{1}, F_{2}, \cdots, F_{n}$ are homogeneous polynomials of positive degrees $d_{1}, d_{2}, \cdots, d_{n}$ in the variables $x_{0}, x_{1}, \cdots, x_{n}$,

$$
F_{i}=\sum_{|\alpha|=d_{i}} c_{i \alpha} x^{\alpha}
$$

Then modulo obvious scalar multiplication of systems, there is a one-to-one correspondence between such systems and points in $\mathbb{P}$.

For such a system, the solution set in $\mathbb{C P}^{n}$ has dimension $\geq n-n=0$. For systems corresponding to generic points in $\mathbb{P}$, the solution sets have dimension 0 , i.e. they have only finitely many solutions in $\mathbb{C P}^{n}$.

Let $X \subset \mathbb{P}$ be the set of points whose corresponding systems have infinitely many solutions in $\mathbb{C P}^{n}$, i.e.,

$$
X=\left\{\left(\left[c_{i, \alpha}\right]\right) \mid F_{1}=\cdots=F_{n}=0 \text { has infinitely many solutions in } \mathbb{C P}^{n}\right\} .
$$

Obviously $\operatorname{codim} \bar{X} \geq 1$ in $\mathbb{P}$, where $\bar{X}$ is the Zariski closure of $X$.

Let us analyze the subset $X$ in more detail. We will use a kind of hidden variable argument similar to [8], and we must proceed carefully in order to apply results on resultants, because the systems should have $n$ homogeneous equations in $n$ variables or $n$ inhomogeneous equations in $n-1$ variables.

Fix a general system $F_{1}=F_{2}=\cdots=F_{n}=0$,

$$
F_{i}=\sum_{|\alpha|=d_{i}} u_{i \alpha} x^{\alpha}
$$

where $\alpha=\left(\alpha_{0}, \alpha_{1}, \cdots, \alpha_{n}\right)$.

For $0 \leq s \neq t \leq n$, we introduce a set of variables $y_{j}, j=0, \cdots, \hat{s}, \cdots, n$ (more precise notation would be $y_{j}^{s, t}$ ). Then in $F_{i}, i=1, \cdots, n$, we replace $x_{j}$ by $y_{j}$ for $j \neq s$, and 
replace $x_{s}$ by $\frac{x_{s}}{x_{t}} y_{t}$. Thus we write $F_{i}$ as a homogeneous polynomial of degree $d_{i}$ in $y_{j}, j \neq s$ with coefficients in $\mathbb{C}\left[\frac{x_{s}}{x_{t}}\right]$ :

$$
\sum_{|\beta|=d_{i}} f_{i, \beta}^{s, t}\left(\frac{x_{s}}{x_{t}}\right) y^{\beta}
$$

$\beta=\left(\beta_{0}, \cdots, \hat{\beta}_{s}, \cdots, \beta_{n}\right)$, and we denote it by $F_{i}^{s, t}$. The set of multi-indices $\alpha$ for $x$-variables with $\alpha_{s}=0$ is in one-to-one correspondence with multi-indices $\beta$ for $y$ variables. Obviously

$$
f_{i, \beta}^{s, t}\left(\frac{x_{s}}{x_{t}}\right)=\sum_{\alpha_{j}=\beta_{j}, j \neq s, t, \alpha_{s}+\alpha_{t}=\beta_{t}} u_{i, \alpha}\left(\frac{x_{s}}{x_{t}}\right)^{\alpha_{s}} .
$$

Let us make this treatment clear by an example. For the homogeneous polynomial $F=x_{0}^{2} x_{1}+x_{0} x_{1}^{2}+x_{1} x_{2}^{2}+x_{1}^{2} x_{2}$, if we take $s=0, t=1$, then we obtain the homogeneous polynomial $\left[\left(\frac{x_{0}}{x_{1}}\right)^{2}+\frac{x_{0}}{x_{1}}\right] y_{1}^{3}+y_{1} y_{2}^{2}+y_{1}^{2} y_{2}$ after the replacement.

The system $F_{1}^{s, t}=\cdots=F_{n}^{s, t}=0$ is a system with $n$ homogeneous equations in $n$ variables, so the resultant $\operatorname{Res}^{s, t}=\operatorname{Res}\left(F_{1}^{s, t}, \cdots, F_{n}^{s, t}\right)$ is a polynomial in variables $f_{i, \beta}^{s, t}$, thus a polynomial in $\mathbb{Z}\left[u_{i, \alpha}, i=1, \cdots n, \frac{x_{s}}{x_{t}}\right]$.

LEMMA 5.1. If the system $F_{1}=F_{2}=\cdots=F_{n}=0$ has a solution $\left(a_{0}, a_{1}, \cdots, a_{n}\right)$ with $a_{t} \neq 0$, then for any $s \neq t, \frac{a_{s}}{a_{t}}$ is a solution of $\operatorname{Res}\left(F_{1}^{s, t}, \cdots, F_{n}^{s, t}\right)=0$.

Proof. The system $\sum_{|\alpha|=d_{i}} f_{i, \alpha}^{s, t}\left(\frac{a_{s}}{a_{t}}\right) y^{\alpha}=0, i=1, \cdots, n$ has a non-trivial solution $\left(\frac{a_{i}}{a_{t}}\right)_{i \neq s}$, so $\frac{a_{s}}{a_{t}}$ is a solution of $\operatorname{Res}\left(F_{1}^{s, t}, \cdots, F_{n}^{s, t}\right)=0$.

Lemma 5.2. For any $\left(c_{i, \alpha}\right) \in X$, at least one of the polynomials $\operatorname{Res}^{s, t}\left(c_{i, \alpha}, \frac{x_{s}}{x_{t}}\right)$ vanished identically.

Proof. Otherwise, a solution $\left(a_{1}, \cdots, a_{n}\right)$ of the system $F_{1}=F_{2}=\cdots=F_{n}=0$ must satisfy $\operatorname{Res}^{s, t}\left(c_{i, \alpha}, \frac{a_{s}}{a_{t}}\right)=0$ for any $t$ with $a_{t} \neq 0$ and $s \neq t$; hence, there can be at most finitely many solutions.

Theorem 5.1. The Zariski closure $\bar{X}$ of $X$ has codimension at least 2 in $\mathbb{P}$ if $n \geq 2$.

Proof. For $0 \leq s \neq t \leq n$, let

$$
X^{s, t}=\left\{\left[c_{i, \alpha}\right] \mid \operatorname{Res}^{s, t}\left(c_{i, \alpha}, \frac{x_{s}}{x_{t}}\right) \equiv 0\right\}
$$

Obviously $\bar{X} \subset \cup X^{s, t}$, so if we can prove that every $X^{s, t}$ has codimension at least 2, then so does $\bar{X}$.

As a polynomial in the variable $\frac{x_{s}}{x_{t}}$ with coefficients in $\mathbb{Z}\left[u_{i, \alpha}, i=1, \cdots n\right]$, the constant term of $\operatorname{Res}^{s, t}\left(u_{i, \alpha}, \frac{x_{s}}{x_{t}}\right)$ is the resultant for the reduced system of our general system by letting $x_{s}=0$, which is a general system in $n$ variables $y_{j}, j \neq s$. We denote the constant term by $\operatorname{Res}\left(u_{i, \alpha}, \alpha_{s}=0\right)$, which by formula (5.1) is an irreducible polynomial in the variables $u_{i, \alpha}$ (with $\alpha_{s}=0$ ),

Now let us consider the coefficient of the linear term in $\operatorname{Res}^{s, t}\left(u_{i, \alpha}, \frac{x_{s}}{x_{t}}\right)$. Notice by formula (5.1) that if we take the degree $\frac{x_{s}}{x_{t}}$ to be 0 , then $\operatorname{Res}^{s, t}\left(u_{i, \alpha}, \frac{x_{s}}{x_{t}}\right)$ is a homogeneous polynomial.

For the coefficient of the linear term, again by formula (5.1) every monomial is a monomial in variables $u_{i, \alpha}$ with $\alpha_{s}=0$ times a variable $u_{i, \alpha}$ where $\alpha_{s}=1$, and hence 
has lower degree in the variables $u_{i, \alpha}$ with $\alpha_{s}=0$, and it has the same degree as the constant term, so it is coprime with constant term (since the constant term is irreducible).

Inside $\mathbb{P}(\operatorname{dim}(\mathbb{P}) \geq 2$ if $n \geq 2)$, the subvariety defined by the constant term and the coefficient of the linear term cannot be the zero set of a single polynomial, so must have codimension $\geq 2$. The subvariety $X^{s, t}$ is contained in this variety, so $X^{s, t}$ has codimension at least 2 .

Up to a common scalar multiplication, the set of hypermatrices is in one-to-one correspondence with points in $\mathbb{C} \mathbb{P}^{n^{m}-1}$.

THEOREM 5.3. In the space $\mathbb{C P}^{n^{m}-1}$, the set of hypermatrices with infinitely many eigenpairs has codimension at least $2(n \geq 2)$.

Proof. Let $A$ be an $m$ th order hypermatrix. Then by an argument similar to that in Theorem 5.1, the subset of hypermatrices $A$ for which $A x^{m-1}=0$ has infinitely many solutions is contained in a subvariety (we denote it by $X_{0}$ ) which is defined by two coprime polynomials, and $X_{0}$ has codimension $\geq 2$, i.e., the closure of the subset for which 0 is an eigenvalue in infinitely many eigenpairs only is a codimension $\geq 2$ subvariety.

Now, the system

$$
A x^{m-1}=\lambda x
$$

it has infinitely many solutions with $\lambda \neq 0$ if and only if

$$
A x^{m-1}=x_{0}^{m-2} x
$$

has infinitely many solutions with $x_{0} \neq 0$.

For $s=1, \cdots n$, we introduce the variable $y=0, \cdots, \hat{y}_{s}, \cdots, y_{n}$, and rewrite the system $A x^{m-1}=x_{0}^{m-2} x$ as follows: replace $x_{j}$ by $y_{j}$ for $j \neq s$, and replace $x_{s}$ by $\frac{x_{s}}{x_{0}} y_{0}$, so that we have a new system $F_{1}^{s}=F_{2}^{s}=\cdots=F_{n}^{s}=0$, which is obtained from $A x^{m-1}=0$ by replacing $x_{j}$ by $y_{j}$ for $j \neq s$ and $x_{s} \mapsto \frac{x_{s}}{x_{0}} y_{0}$, and by a translation of coefficients, with an extra term $-y_{0}^{m-2} y_{i}$ for $i \neq s$ and $-\frac{x_{s}}{x_{0}} y_{0}^{m-1}$ for $i=s$.

The resultant $\operatorname{Res}^{s}\left(a_{i_{1} \cdots i_{n}}, \frac{x_{s}}{x_{t}}\right)$ of the system $F_{1}^{s}=F_{2}^{s}=\cdots=F_{n}^{s}=0$ is a polynomial in $\mathbb{Z}\left[a_{i_{1} i_{2} \cdots i_{n}}, \frac{x_{s}}{x_{0}}\right]$ : let

$$
X^{s}=\left\{[A] \mid \operatorname{Res}^{s}\left(a_{i_{1} \cdots i_{n}}, \frac{x_{s}}{x_{t}}\right) \equiv 0\right\} \subset \mathbb{C P}^{n^{m}-1}
$$

Then by an argument similar to that in the proof of Theorem 5.1, the set of $A$ for which $A x^{m-1}=x_{0}^{m-2} x$ has infinitely many solutions with $x_{0} \neq 0$ is a subset of $\cup_{s=1}^{n} X^{s}$, and $X^{s}$ has codimension at least 2 .

Thus, the set of hypermatrices with infinitely many eigenpairs is a subset of the subvariety $X_{0} \cup_{s=1}^{n} X^{s}$. Hence its closure has codimension $\geq 2$.

\section{A bound on the codimension of the variety of irregular tensors}

Let

$$
X=\{[A] \mid A \text { is irregular }\} \subset \mathbb{C P}^{n^{m}-1} .
$$


Theorem 6.1. The Zariski closure $\bar{X}$ of $X$ has codimension at least 2.

Proof. For a tensor $A$, let

$$
F_{i}=\sum a_{i i_{2} \cdots i_{m}} x_{i_{2}} \cdots x_{i_{m}} .
$$

Then $A$ is irregular if and only if

$$
F_{1}=F_{2}=\cdots=F_{n}=x^{T} x=0
$$

has a solution in $\mathbb{C P}^{n-1}$, since this is a system with $n+1$ homogeneous equations in $n$ variables.

Now assume $A$ is irregular; then for any $i=1, \cdots, n$, the system

$$
F_{1}=F_{2}=\cdots=\hat{F}_{i}=\cdots=F_{n}=x^{T} x=0
$$

has a nontrivial solution, and therefore the resultant $\Delta_{i}$ of this system is 0 .

For a hypermatrix $A$ with only nontrivial entries $a_{j j \cdots j j}=1$, the system

$$
F_{1}=F_{2}=\cdots=\hat{F}_{i}=\cdots=F_{n}=x^{T} x=0
$$

is

$$
\left\{\begin{array}{l}
x_{j}^{m-1}=0, j=1, \cdots, \hat{i}, \cdots, n, \\
x^{T} x=0
\end{array}\right.
$$

and has no solution in $\mathbb{C P}^{n-1}$, so $\Delta_{i} \not \equiv 0$.

Notice for the resultant $\Delta_{i}$, the variables $a_{i i_{2} \cdots i_{n}}$ are missing, so the greatest common divisor of $\Delta_{1}, \cdots, \Delta_{n}$ is 1 . i.e, any component of the subvariety of $\mathbb{C P} \mathbb{P}^{m}-1$ defined by

$$
\Delta_{1}=\cdots=\Delta_{n}=0
$$

cannot be defined by a single polynomial. Hence any component of the subvariety $\Delta_{1}=\cdots=\Delta_{n}=0$ has codimension $\geq 2$.

Now we have the conclusion because

$$
\bar{X} \subset\left\{\Delta_{1}=\cdots=\Delta_{n}=0\right\} .
$$

\section{The leading coefficient}

We now study the properties of the leading coefficient $a_{h(m, n)}(m, n)$ by exploring the differences between the definitions of E-eigenvalues and eigenpair equivalence classes. We see that besides those eigenpair equivalence classes which correspond to E-eigenvalues, all of the other eigenpair equivalence classes correspond to nonzero solutions of

$$
\left\{\begin{array}{c}
A x^{m-1}=\lambda x \\
x^{T} x=0
\end{array}\right.
$$

We call (7.1) the deficit system of $A$.

Now we investigate the leading coefficient for the characteristic polynomials for tensors when $n=2$. 
Lemma 7.1. For a regular tensor, the leading coefficient of its characteristic polynomial is 0 if and only if the system

$$
\left\{\begin{array}{l}
A x^{m-1}=\lambda x \\
x^{T} x=0
\end{array}\right.
$$

has a nontrivial solution.

Proof. Consider the inhomogeneous system

$$
\left\{\begin{array}{l}
A x^{m-1}=\lambda x \\
x^{T} x=1
\end{array}\right.
$$

This system has no solution at $\infty$ because of regularity.

If we consider $\lambda$ as a constant and homogenize the system with respect to $x_{0}$, we have the following system:

$$
\left\{\begin{array}{l}
A x^{m-1}-\lambda x_{0}^{m-2} x=0 \\
x^{T} x-x_{0}^{2}=0
\end{array}\right.
$$

The resultant for this homogeneous system (the E-characteristic polynomial) has the exact information of multiplicity of eigenpairs, i.e., it is a constant times [8]

$$
\Pi_{\left(\lambda_{i}, x_{i}\right)}\left(\lambda-\lambda_{i}\right)^{m\left(\lambda_{i}, x_{i}\right)},
$$

where the product is over distinct eigenpairs $\left\{\left(\lambda_{i}, x_{i}\right)\right\}$, and $m\left(\lambda_{i}, x_{i}\right)$ is its multiplicity.

Therefore, if the leading term is 0 , there is some eigenpair missing, which must be a solution of

$$
\left\{\begin{array}{l}
A x^{m-1}=\lambda x \\
x^{T} x=0
\end{array}\right.
$$

On the other hand, if the leading term is not 0 , the deficit system cannot have a nontrivial solution, otherwise $A$ will have more eigenpairs than expected.

LEMma 7.2. For a regular tensor, the system

$$
\left\{\begin{array}{l}
A x^{m-1}=\lambda x \\
x^{T} x=0
\end{array}\right.
$$

has a nontrivial solution if and only if

$$
P_{m}^{2}+Q_{m}^{2}=0,
$$

where $P_{m}$ and $Q_{m}$ are the sum of the first $m$ terms of the following two series respectively:

$$
b_{1}-c_{2}-b_{3}+c_{4}+b_{5}-c_{6}-b_{7}+c_{8}+b_{9}-c_{10}-\cdots
$$

and

$$
c_{1}+b_{2}-c_{3}-b_{4}+c_{5}+b_{6}-c_{7}-b_{8}+c_{9}+b_{10}-\cdots,
$$

where the signs of these terms are cyclically changed with the cycle four, and $b_{j}=$ $\sum\left\{a_{1 i_{2} \cdots i_{m}}\right.$ : exactly $j-1$ of $i_{2}, \cdots, i_{m}$ are 2$\}$ for $j=1, \cdots, m$, and $c_{j}=\sum\left\{a_{2 i_{2} \cdots i_{m}}\right.$ : exactly $j-1$ of $i_{2}, \cdots, i_{m}$ are 2$\}$ for $j=1, \cdots, m$. 
Proof. Since $n=2$, if the deficit system (7.1) has a nontrivial solution, then all the nontrivial solutions of the deficit system (7.1) are nonzero multiples of $(1, \sqrt{-1})$ or $(1,-\sqrt{-1})$. Therefore, there are at most two eigenpair equivalence classes corresponding to nonzero solutions of the deficit system (7.1) when $n=2$. Note that when $n=2, A x^{m-1}=\lambda x$ can be written as

$$
\left\{\begin{array}{l}
\sum_{i=1}^{m} b_{i} x_{1}^{m-i} x_{2}^{i-1}=\lambda x_{1}, \\
\sum_{i=1}^{m} c_{i} x_{1}^{m-i} x_{2}^{i-1}=\lambda x_{2} .
\end{array}\right.
$$

Substituting $(1, \sqrt{-1})$ to $(7.2)$, we have

$$
\left\{\begin{array}{l}
\sum_{i=1}^{m} b_{i}(\sqrt{-1})^{i-1}=\lambda, \\
\sum_{i=1}^{m} c_{i}(\sqrt{-1})^{i-1}=\lambda \sqrt{-1} .
\end{array}\right.
$$

Eliminating $\lambda(\lambda \neq 0$ by regularity), we have

$$
\sum_{i=1}^{m} b_{i}(\sqrt{-1})^{i-1}+\sum_{i=1}^{m} c_{i}(\sqrt{-1})^{i}=0 .
$$

By the definitions of $P_{m}$ and $Q_{m}$, we have $P_{m}=-Q_{m} \sqrt{-1}$. Similarly, substituting $(1, \sqrt{-1})$ to $(7.2)$ and eliminating $\lambda$, we have $P_{m}=Q_{m} \sqrt{-1}$. These imply $P_{m}^{2}+Q_{m}^{2}=0$.

On the other hand, if $P_{m}^{2}+Q_{m}^{2}=0$, by a similar but reverse argument, we see that $(1, \sqrt{-1})$ or $(1,-\sqrt{-1})$ is a nontrivial solution of the deficit system (7.1). This completes the proof.

THEOREM 7.3. If A has only finitely many equivalence classes of eigenpairs, then the leading coefficient $a_{h(m, 2)}(m, 2)=0$ if and only if

$$
P_{m}^{2}+Q_{m}^{2}=0 .
$$

Proof. First, if $A$ has only finitely many equivalence classes of eigenpairs, then its characteristic polynomial cannot be identically 0 .

Let us consider the following subvarieties of $\mathbb{C P}^{2^{m}-1}: \quad X=\left\{a_{h(m, 2)}(m, 2)=\right.$ $0\}$, and $Y=\left\{P_{m}^{2}+Q_{m}^{2}=0\right\}$, which both have codimension 1. Let $Z_{1}=$ $\{[A] \mid \mathrm{A}$ has infinitely many eigenpairs $\}$, and $Z_{2}=\{[A] \mid A$ is irregular $\}$. Then both $Z_{1}$ and $Z_{2}$ have codimension $\geq 2$. So $X-\bar{Z}_{1}-\bar{Z}_{2}$ and $Y-\bar{Z}_{1}-\bar{Z}_{2}$ are not empty.

By Lemmas 7.1 and $7.2, X-\bar{Z}_{1}-\bar{Z}_{2} \subset Y$ and $Y-\bar{Z}_{1}-\bar{Z}_{2} \subset X$, so that by taking closure we have $X \subset Y$ and $Y \subset X$. This implies that $X=Y$ and the conclusion follows.

口

THEOREM 7.4. If A has only finitely many equivalence classes of eigenpairs, then the leading coefficient $a_{h(m, 2)}(m, 2)$ is

$$
\left(P_{m}^{2}+Q_{m}^{2}\right)^{\frac{m-2}{2}}
$$

for even $m$, and

$$
-\left(P_{m}^{2}+Q_{m}^{2}\right)^{m-2}
$$

for odd $m$. 
Proof. Let $W=\left\{[B] \mid P_{m}^{2}+Q_{m}^{2}=0\right\} . W$ can be written as a union of two irreducible varieties

$$
W=W_{1} \bigcup W_{2},
$$

where

$$
\begin{aligned}
& W_{1}=\left\{[B] \mid P_{m}+\sqrt{-1} Q_{m}=0\right\}, \\
& W_{2}=\left\{[B] \mid P_{m}-\sqrt{-1} Q_{m}=0\right\} .
\end{aligned}
$$

Since $P_{m}+\sqrt{-1} Q_{m}$ and $P_{m}-\sqrt{-1} Q_{m}$ are irreducible, by Theorem 7.3

$$
a_{h(m, 2)}(m, 2)=\left(P_{m}+\sqrt{-1} Q_{m}\right)^{k}\left(P_{m}-\sqrt{-1} Q_{m}\right)^{s} C,
$$

where $C$ is a constant. Since $a_{h(m, 2)}(m, 2) \in \mathbb{Z}\left[a_{i_{1} \cdots i_{m}}\right], k$ must be equal to $s$. Then by checking the degree, we see that our results hold up to a scaling constant. By checking an example such as $a_{1 \cdots 1}=a_{2 \cdots 2}=1$ but all the other entries of $A$ are zero, we have the results.

Acknowledgment. We are thankful to the two referees, whose comments greatly improved our paper.

\section{REFERENCES}

[1] V. Balan, Spectral properties and applications of numerical multi-linear algebra of m-root structures, in: Hypercomplex Numbers in Geometry and Physics, ed., "Mozet", Russia, 2, 101$107,2008$.

[2] V. Balan, Numerical multilinear algebra of symmetric m-root structures: Spectral properties and applications, Symmetry: Culture and Science, Symmetry Festival, 2009, Symmetry in the History of Science, Art and Technology; Part 2; Geometric Approaches to Symmetry, 21, 119-131, 2010.

[3] V. Balan, Spectra of symmetric tensors and m-root Finsler models, Lin. Alg. Appl., 436, 152$162,2012$.

[4] V. Balan and N. Perminov, Applications of resultants in the spectral M-root framework, Appl. Sci., 12, 20-29, 2010.

[5] L. Bloy and R. Verma, On computing the underlying fiber directions from the diffusion orientation distribution function, in: Medical Image Computing and Computer-Assisted Intervention - MICCAI 2008, D. Metaxas, L. Axel, G. Fichtinger and G. Székeley eds., Springer-Verlag, Berlin, 1-8, 2008.

[6] D. Cartwright and B. Sturmfels, The number of eigenvalues of a tensor, Lin. Alg. Appl., to appear.

[7] K.C. Chang, K. Pearson, and T. Zhang, On eigenvalues of real symmetric tensors, J. Math. Anal. Appl., 350, 416-422, 2009.

[8] D. Cox, J. Little, and D. O'Shea, Using Algebraic Geometry, Springer-Verlag, New York, 1998.

[9] S. Hu and L. Qi, Algebraic connectivity of an even uniform hypergraph, J. Comb. Opt., 2011.

[10] I.M. Gelfand, M.M. Kapranov, and A.V. Zelevinsky, Discrimants, Resultants and Multidimensional Determinants, Birkhäuser, Boston, 1994.

[11] J.J. Hilling and A. Sudbery, The geometric measure of multipartite entanglement and the singular values of a hypermatrix, J. Math. Phys., 51, 072102, 2010.

[12] T.G. Kolda and J.R. Mayo, Shifted power method for computing tensor eigenpairs, SIAM J. Matrix Anal. Appl., 32, 1095-1124, 2011.

[13] L-H. Lim, Singular values and eigenvalues of tensors: A variational approach, Proceedings of the First IEEE International Workshop on Computational Advances in Multi-Sensor Adaptive Processing (CAMSAP), December 13-15, 129-132, 2005.

[14] G. Ni, L. Qi, F. Wang, and Y. Wang, The degree of the E-characteristic polynomial of an even order tensor, J. Math. Anal. Appl., 329, 1218-1229, 2007. 
[15] L. Qi, Eigenvalues of a real supersymmetric tensor, J. Symbolic Comput., 40, 1302-1324, 2005.

[16] L. Qi, Rank and eigenvalues of a supersymmetric tensor, a multivariate homogeneous polynomial and an algebraic surface defined by them, J. Symbolic Comput., 41, 1309-1327, 2006.

[17] L. Qi, Eigenvalues and invariants of tensors, J. Math. Anal. Appl., 325, 1363-1377, 2007.

[18] L. Qi, The best rank-one approximation ratio of a tensor space, SIAM J. Matrix Anal. Appl., 32, 430-442, 2011.

[19] L. Qi, F. Wang, and Y. Wang, Z-eigenvalue methods for a global polynomial optimization problem, Math. Prog., 118, 301-316, 2009.

[20] L. Qi, G. Yu, and E.X. Wu, Higher order positive semi-definite diffusion tensor imaging, SIAM J. Imag. Sci., 3, 416-433, 2010.

[21] S. Ragnarsson and C.F. van Loan, Block tensors and symmetric embeddings, Lin. Alg. Appl., to appear.

[22] T.C. Wei and P.M. Goldbart, Geometric measure of entanglement and applications to bipartite and multipartite quantum states, Phys. Rev. A, 68, 042307, 2003.

[23] T. Zhang, Existence of real eigenvalues of real tensor, Nonlin. Anal., 74, 2862-2868, 2011.

[24] X. Zhang, L. Qi, and Y. Ye, The cubic spherical optimization problem, Math. Comput., 81, 1513-1525, 2012. 